\title{
Pulmonary fat embolism and related effects during femoral intramedullary surgery: An experimental study in dogs
}

\author{
FEIHU ZHOU ${ }^{1}, \mathrm{JUN} \mathrm{JI}^{2}$, QING SONG ${ }^{1 *}, \mathrm{ZHIYONG} \mathrm{PENG}^{3}$, GUOQIANG ZHANG $^{4}$ and YAN WANG ${ }^{4 *}$ \\ ${ }^{1}$ Department of Critical Care Medicine, Chinese People's Liberation Army General Hospital, Beijing 100853; \\ ${ }^{2}$ Department of Anesthesiology, Air Force General Hospital, Beijing 100142, P.R. China; \\ ${ }^{3}$ The Clinical Research, Investigation, and Systems Modeling of Acute Illness (CRISMA) Center, \\ Department of Critical Care Medicine, University of Pittsburgh, Pittsburgh, PA, USA; \\ ${ }^{4}$ Department of Orthopaedics, Chinese People's Liberation Army General Hospital, Beijing 100853, P.R. China
}

Received February 20, 2013; Accepted May 21, 2013

DOI: $10.3892 /$ etm.2013.1143

\begin{abstract}
The aim of the present study was to develop an animal model of pulmonary fat embolism (PFE) caused by femoral intramedullary procedures, and to investigate the initial changes in the hemodynamics, cytokines and risk factors of PFE. Sixteen dogs were randomly divided into two groups: Group A (intramedullary reaming and bone cement injection, $n=8$ ) and Group B (surgical approach without opening the medullary cavity, $n=8$ ). The hemodynamics, arterial blood gases and relevant cytokines were evaluated, and the lungs were examined using Oil Red O staining. In the animals of Group A, the heart rate, central venous pressure, mean pulmonary arterial pressure, pulmonary capillary wedge pressure and extravascular lung water (EVLW) were increased compared with the baseline levels, while the mean arterial pressure was decreased immediately following the reaming and bone cement infusion $(\mathrm{P}<0.05)$. Furthermore, there was a significant reduction in the $\mathrm{pH}$ and the arterial oxygen tension $\left(\mathrm{PaO}_{2}\right)$, and a significant increase in the arterial carbon dioxide tension $\left(\mathrm{PaCO}_{2} ; \mathrm{P}<0.05\right.$ for all) following the bilateral intramedullary surgery. The EVLW was correlated with the $\mathrm{PaO}_{2}(\mathrm{P}<0.001)$ and $\mathrm{PaCO}_{2}(\mathrm{P}=0.046)$. Following surgery, there was a significant increase in tumor necrosis factor- $\alpha$ (TNF- $\alpha)$, interleukin-1 $\beta$ (IL-1 $\beta$ ) and IL- 6 levels in Group A $(\mathrm{P}<0.05)$. However, there were no significant changes in these parameters in Group B. The parameters tested, with the exception of $\mathrm{pH}$, were significantly different in Group A compared with those in Group B $(\mathrm{P}<0.05)$ following the
\end{abstract}

Correspondence to: Professor Yan Wang, Department of Orthopaedics, Chinese People's Liberation Army General Hospital, 28 Fuxing Road, Beijing 100853, P.R. China

E-mail: fhdoccn@163.com

${ }^{*}$ Contributed equally

Key words: pulmonary fat embolism, hemodynamics, cytokine, extravascular lung water bilateral intramedullary surgery. Oil Red O staining was positive for all animals in Group A and negative for those in Group B. Femoral intramedullary surgery may induce PFE and subsequently affect hemodynamics and arterial blood gases. EVLW was correlated with the $\mathrm{PaO}_{2}(\mathrm{P}<0.001)$ and the $\mathrm{PaCO}_{2}(\mathrm{P}=0.046)$. These results demonstrated that EVLW and cytokines may serve as predictors of the development of fat embolism syndrome (FES).

\section{Introduction}

Fat embolism syndrome (FES) is a severe complication of orthopedic surgery or trauma. It occurs in $\sim 1.29 \%$ of patients with multiple fractures, particularly those with a femoral fracture (1). Typically, it triggers the development of pulmonary fat embolism (PFE), which has been reported to be present in $82 \%$ of trauma patients (2). The acute consequences of fat embolism are hemodynamic disorders and, in severe cases, right-sided heart failure or hypoxemia $(3,4)$. Intraoperative cardiovascular deterioration, as a result of the pulmonary embolization of bone marrow fat, is a potential complication (5). Although numerous animal models that reveal many aspects of PFE have been developed (5-9), the underlying mechanisms of the syndrome, including the early hemodynamic effects, the potential inflammatory responses and the risk factors of PFE, are not fully understood. The aim of the present study was to develop a clinically relevant animal model, and to investigate the initial changes in the hemodynamics, cytokines, arterial blood gases and the risk factors involved in PFE that are caused by femoral intramedullary procedures.

\section{Subjects and methods}

Subjects. The study was approved by the Animal Research Committee of the Chinese People's Liberation Army (PLA) General Hospital (Beijing, China). Sixteen healthy male outbred dogs (weight, 14.7-22.3 kg), were randomly divided into two groups: Group A (intramedullary reaming and bone cement injection, $\mathrm{n}=8$ ) and Group $\mathrm{B}$ (surgical approach without opening the medullar cavity, $n=8$ ). 


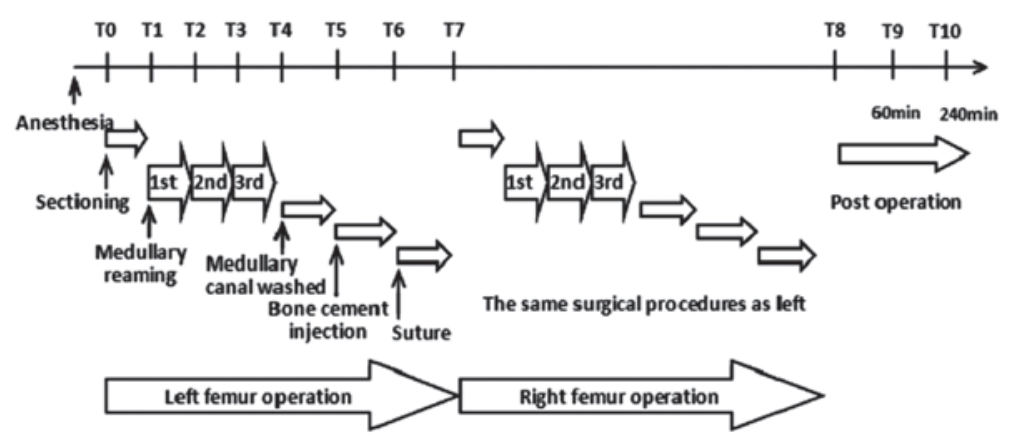

Figure 1. Surgical approaches and associated time points. T0, prior to surgery; T1, prior to unilateral medullary reaming; T2, following the first unilateral medullary reaming; T3, following the second unilateral medullary reaming; T4, following the third unilateral medullary reaming; T5, prior to unilateral bone cement injection; T6, 5 min following unilateral bone cement injection; T7, following unilateral femur surgery; T8, following bilateral femur surgery; T9, 60 min following bilateral femur surgery; T10, 240 min following bilateral femur surgery.

Surgical procedure and measurement of biochemical parameters. Anesthesia was induced with an intravenous injection of sodium pentobarbital $(30 \mathrm{mg} / \mathrm{kg})$, and maintained with a continuous infusion $(5 \mathrm{mg} / \mathrm{kg} / \mathrm{min})$. Each dog was orally intubated with an endotracheal tube, and mechanically ventilated with a Servo-i Ventilator [Maquet, Inc., Rastatt, Germany; basic setting: volume control ventilation, initial rate $18 / \mathrm{min}$ and tidal volume $12 \mathrm{ml} / \mathrm{kg}$; I:E=1:2; positive end expiratory pressure (PEEP), $4 \mathrm{~cm} \mathrm{H} \mathrm{H}_{2} \mathrm{O}$; and $\mathrm{FiO}_{2}, 40 \%$ ] during the experiment. A pulmonary artery catheter (Arrow International, Inc., Reading, PA, USA) was inserted through the external jugular vein. A pulse contour cardiac output (PiCCO) hot dilated catheter was inserted via femoral cut-down and connected to the PiCCO monitor (PiCCO Plus, Pulsion Medical System AG, Munich, Germany) for the determination of pulmonary and systemic hemodynamics. The biochemical parameters, including hemodynamic parameters, arterial blood gases and cytokine levels, were measured. Hemodynamic data were recorded at specific time points, and blood samples were collected for the measurement of arterial blood gases and cytokine levels at such time points (Fig. 1). The biochemical parameters comprised heart rate (HR), mean arterial pressure (MAP), central venous pressure (CVP), mean pulmonary arterial pressure (MPAP), pulmonary capillary wedge pressure (PCWP) and extravascular lung water (EVLW). In the arterial blood gas tests, the $\mathrm{pH}$, arterial oxygen tension $\left(\mathrm{PaO}_{2}\right)$ and arterial carbon dioxide tension $\left(\mathrm{PaCO}_{2}\right)$ were evaluated. In addition, the cytokine levels that were measured were those of tumor necrosis factor- $\alpha$ (TNF- $\alpha$ ), interleukin- $1 \beta$ (IL-1 $\beta$ ) and IL-6. The time points at which measurements were taken were as follows: T0, prior to surgery; T1, prior to unilateral medullary reaming; $\mathrm{T} 2$, following the first unilateral medullary reaming; $\mathrm{T} 3$, following the second unilateral medullary reaming; T4, following the third unilateral medullary reaming; $\mathrm{T} 5$, prior to unilateral bone cement injection; T6, 5 min following unilateral bone cement injection; $\mathrm{T} 7$, following unilateral femur surgery; T8, following bilateral femur surgery; T9, $60 \mathrm{~min}$ following bilateral femur surgery; T10, 240 min following bilateral femur surgery.

In Group A, the greater lateral trochanter of the left femur was resected through a muscle gap by the posterior lateral femur approach. The intertrochanteric fossa and entry of the medullary canal were exposed and followed by intramedullary reaming to one-half the length of the femur. The medullary canal was washed with saline to remove destroyed myeloid tissues, and dried with gauze. Bone cement (Tianjin Synthetic Material Research Institute, Hexi, China) was prepared by manually stirring the powder into the solution at a ratio of $2: 1$, until a dough was formed, which was then used to fill the medullary canal. When completely solidified, the incision was sutured. The same surgical approaches were repeated in the right femurs of the dogs in Group A. The femurs of the dogs in Group B were subjected to the same surgical methods as those in Group A, with the exception of the resection of the greater trochanter and the opening of the medullary cavity. The anesthetized dogs were sacrificed by the intravenous injection of $5 \mathrm{ml}$ potassium chloride (15 meq) $240 \mathrm{~min}$ following bilateral femur surgery. Necropsies were performed immediately following sacrifice, to obtain the right and left lungs. Oil Red O staining was used to identify the presence of fatty deposits in these tissues.

Statistical analysis. Data are presented as the mean \pm standard deviation. The mean differences between the study groups and within groups among different time points were analyzed by analysis of variance (ANOVA) for repeated measures. Correlations between variables were analyzed and expressed as correlation coefficients. $\mathrm{P}<0.05$ was considered to indicate a statistically significant difference. The statistical analysis was performed using SPSS 13.0 for Windows (SPSS, Inc., Chicago, IL, USA).

\section{Results}

All animals survived the experimental procedure. In all lung sections from Group A, fat emboli stained by Oil Red O were observed, which demonstrated that the animals had developed PFE (Fig. 2A). However, no fat emboli were identified in any of the lung sections from Group B (Fig. 2B).

Figs. $3 \mathrm{~A}$ and $\mathrm{B}$ demonstrate the changes in the hemodynamics in Group A. The HR, CVP, MPAP, PCWP and EVLW increased, while the MAP decreased, immediately following the reaming and bone cement infusion, compared with those values at $\mathrm{T} 0$. In addition, the values at $\mathrm{T} 4$ and $\mathrm{T} 6$ were significantly different from those at $\mathrm{T} 0(\mathrm{P}<0.05)$. Changes in the HR, MAP and PCWP were observed at T10; the values 
A

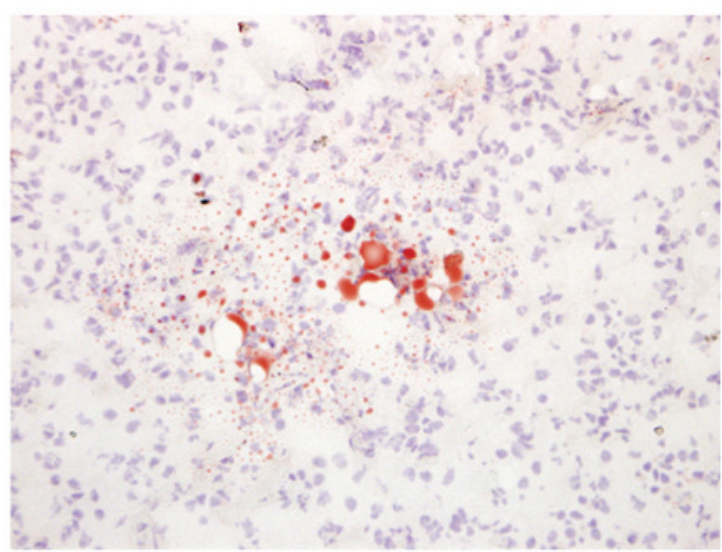

B

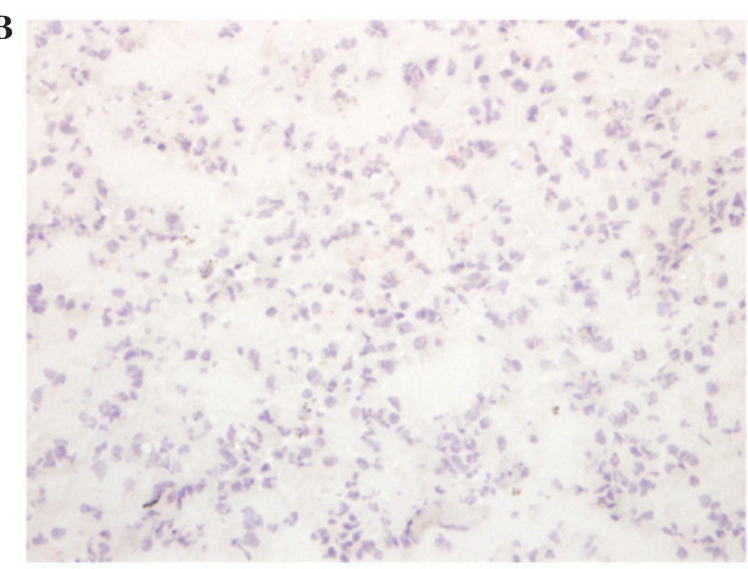

Figure 2. Lung sections of animals from (A) Group A and (B) Group B (A) Shows multiple fat emboli stained by Oil Red O. (B) No staining indicated. Original magnification, $\mathrm{x} 40$.

of the parameters were no longer significantly different from those at $\mathrm{T} 0(\mathrm{P}>0.05)$. By contrast, the CVP, MPAP and EVLW remained high at this time point, compared with those at $\mathrm{T} 0$ $(\mathrm{P}<0.05)$. However, there were no significant differences in the hemodynamics in Group B ( $>0.05)$. Significant differences were identified in the HR, MAP, and PCWP at T8, as well as in the CVP, MPAP and EVLW at T8, T9 and T10 in Group A (Table I), compared with those in Group B.

Group A demonstrated reductions in the $\mathrm{pH}$ and the $\mathrm{PaO}_{2}$, but an increase in the $\mathrm{PaCO}_{2}$, at T8, which were significantly different compared with those at T0 $(\mathrm{P}<0.05$; Table II). Although there was an increase in the $\mathrm{pH}$ and the $\mathrm{PaO}_{2}$, and a reduction in the $\mathrm{PaCO}_{2}$, at T10, the $\mathrm{PaCO}_{2}(46.6 \pm 7.5 \mathrm{mmHg})$ remained high and was significantly different compared with that at $\mathrm{T0}(\mathrm{P}<0.05)$. The $\mathrm{PaO}_{2}$ was correlated with the EVLW [linear correlation coefficient, $-0.5664 ; 95 \%$ confidence interval (CI), -12.181 to $-3.614 ; \mathrm{P}<0.001]$. Similar results were identified between $\mathrm{PaCO}_{2}$ and EVLW (linear correlation coefficient, 0.3560; 95\% CI, 0.02409-2.216; $\mathrm{P}=0.046$ ). However, no significant changes were observed in the arterial blood gases in Group B. Significant differences were identified in the $\mathrm{PaCO}_{2}$ at T8, as well as in the $\mathrm{PaO}_{2}$ at T7 and T8, between Group B and Group A (Table II).

Following the surgery, there were increases in the TNF- $\alpha$, IL-1 $\beta$ and IL-6 levels in Group A. Significant differences were observed in the TNF- $\alpha$ levels at T8 and T10, as well as in the IL- $1 \beta$ and IL- 6 levels at T8 and T10, compared with those at
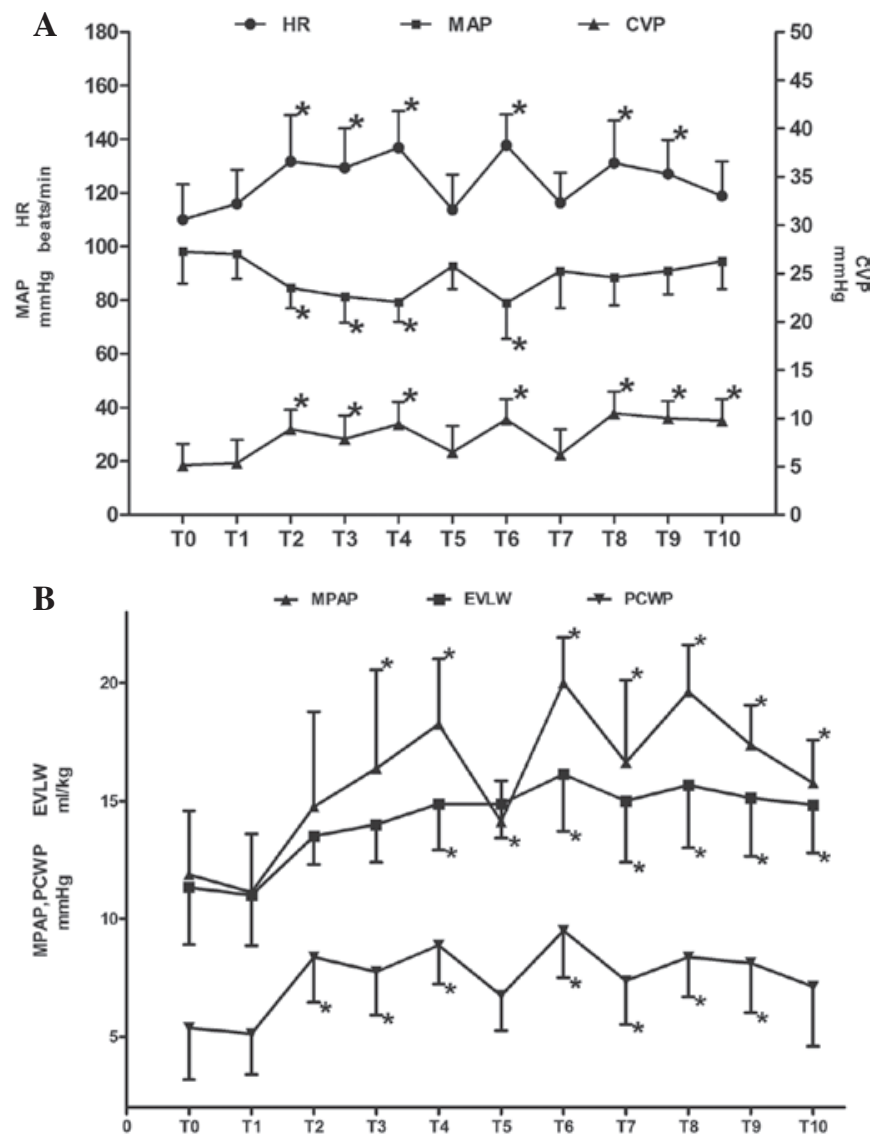

Figure 3. Alterations in the hemodynamic paramters at various time points during and following intramedullary surgery in Group A. (A) Changes in heart rate (HR), mean arterial pressure (MAP) and central venous pressure (CVP). (B) Changes in mean pulmonary arterial pressure (MPAP), extravascular lung water (EVLW) and pulmonary capillary wedge pressure (PCWP). ${ }^{*} \mathrm{P}<0.05$ vs. T0. T0, prior to surgery; $\mathrm{T} 1$, prior to unilateral medullary reaming; T2, following the first unilateral medullary reaming; T3, following the second unilateral medullary reaming; T4, following the third unilateral medullary reaming; T5, prior to unilateral bone cement injection; T6, 5 min following unilateral bone cement injection; T7, following unilateral femur surgery; T8, following bilateral femur surgery; T9, 60 min following bilateral femur surgery; T10, 240 min following bilateral femur surgery.

T0 ( $\mathrm{P}<0.05$; Fig. 4). However, there were no significant differences in the levels of these cytokines in Group B. There were significant differences in the TNF- $\alpha$ and IL- $1 \beta$ levels at T8, as well as in the IL-6 levels at T8 and T10, in Group A, when compared with those in Group B ( $\mathrm{P}<0.05$; Fig. 4).

\section{Discussion}

The present study demonstrated that femoral intramedullary surgery may induce PFE and subsequently affect hemodynamics and arterial blood gases. The EVLW was correlated with the $\mathrm{PaO}_{2}$ and the $\mathrm{PaCO}_{2}$, and may serve as a predictor of the development of FES. Cytokines were significant during these procedures, and may be further predictors for the development of FES. To the best of our knowledge, this study is the first to demonstrate a correlation between the EVLW, cytokines and FES, providing potential methods for the diagnosis and treatment of FES.

The clinical manifestation of PFE ranges from no impairment, in mild cases, to death, in severe cases (4). As for the 
Table I. Hemodynamic data summary for Groups A and B.

\begin{tabular}{|c|c|c|c|c|c|c|c|}
\hline Parameter & Group & T0 & $\mathrm{T} 1$ & $\mathrm{~T} 7$ & $\mathrm{~T} 8$ & T9 & T10 \\
\hline \multirow[t]{2}{*}{ HR (beats/min) } & A & $110.13 \pm 13.18$ & $116.00 \pm 12.71$ & $116.38 \pm 11.17$ & $131.13 \pm 15.91$ & $127.13 \pm 12.68$ & $118.88 \pm 12.84$ \\
\hline & B & $115.63 \pm 12.93$ & $117.13 \pm 10.30$ & $118.38 \pm 9.10$ & $116.13 \pm 7.86^{\mathrm{a}}$ & $120.62 \pm 10.41$ & $117.50 \pm 10.18$ \\
\hline \multirow[t]{2}{*}{ MAP (mmHg) } & A & $98.13 \pm 11.79$ & $97.25 \pm 9.32$ & $90.88 \pm 13.71$ & $88.50 \pm 10.41$ & $91.00 \pm 8.72$ & $94.63 \pm 10.42$ \\
\hline & B & $100.13 \pm 9.33$ & $97.75 \pm 9.22$ & $98.00 \pm 6.01$ & $101.25 \pm 6.34^{\mathrm{a}}$ & $99.38 \pm 6.25$ & $101.38 \pm 5.73$ \\
\hline \multirow[t]{2}{*}{$\mathrm{CVP}(\mathrm{mmHg})$} & A & $5.13 \pm 2.23$ & $5.38 \pm 2.39$ & $6.25 \pm 2.60$ & $10.50 \pm 2.27$ & $10.00 \pm 1.77$ & $9.75 \pm 2.25$ \\
\hline & B & $5.12 \pm 1.36$ & $5.00 \pm 1.76$ & $4.88 \pm 1.13$ & $5.25 \pm 1.28^{\mathrm{a}}$ & $5.50 \pm 1.51^{\mathrm{a}}$ & $5.38 \pm 1.19^{\mathrm{a}}$ \\
\hline \multirow[t]{2}{*}{ MPAP (mmHg) } & A & $11.88 \pm 2.70$ & $11.13 \pm 2.47$ & $16.63 \pm 3.50$ & $19.63 \pm 1.99$ & $17.38 \pm 1.69$ & $15.75 \pm 1.83$ \\
\hline & B & $10.88 \pm 2.90$ & $10.63 \pm 1.85$ & $11.13 \pm 2.99$ & $12.12 \pm 2.18^{\mathrm{a}}$ & $11.37 \pm 2.50^{\mathrm{a}}$ & $11.87 \pm 3.04^{\mathrm{a}}$ \\
\hline \multirow[t]{2}{*}{ PCWP (mmHg) } & A & $5.38 \pm 2.20$ & $5.13 \pm 1.73$ & $7.38 \pm 1.85$ & $8.37 \pm 1.69$ & $8.13 \pm 2.10$ & $7.12 \pm 2.53$ \\
\hline & B & $5.50 \pm 2.45$ & $5.63 \pm 2.20$ & $5.38 \pm 1.19$ & $5.75 \pm 1.04^{\mathrm{a}}$ & $6.13 \pm 1.36$ & $6.63 \pm 1.41$ \\
\hline \multirow[t]{2}{*}{ EVLW (ml/kg) } & A & $11.33 \pm 2.42$ & $11.00 \pm 2.14$ & $15.00 \pm 2.61$ & $15.67 \pm 2.66$ & $15.13 \pm 2.47$ & $14.83 \pm 2.04$ \\
\hline & B & $11.13 \pm 3.48$ & $10.50 \pm 1.77$ & $11.25 \pm 2.66^{\mathrm{a}}$ & $10.75 \pm 2.49^{\mathrm{a}}$ & $10.63 \pm 1.99^{\mathrm{a}}$ & $11.37 \pm 1.77^{\mathrm{a}}$ \\
\hline
\end{tabular}

${ }^{\mathrm{a}} \mathrm{P}<0.05$ indicates a significant difference between Groups A and B. T0, prior to surgery; T1, prior to unilateral medullary reaming; T7, following unilateral femur surgery; T8, following bilateral femur surgery; T9, 60 min following bilateral femur surgery; T10, 240 min following bilateral femur surgery. HR, heart rate; MAP, mean arterial pressure; CVP, central venous pressure; MPAP, mean pulmonary arterial pressure; PCWP, pulmonary capillary wedge pressure; EWLW, extravascular lung water.

Table II. Parameters of arterial blood gases analyzed at different time points in Groups A and B.

\begin{tabular}{lccccc}
\hline Parameter & Group & T0 & T7 & T8 & T10 \\
\hline $\mathrm{pH}$ & $\mathrm{A}$ & $7.420 \pm 0.075$ & $7.370 \pm 0.062$ & $7.336 \pm 0.072^{\mathrm{a}}$ & $7.360 \pm 0.065$ \\
& $\mathrm{~B}$ & $7.399 \pm 0.068$ & $7.414 \pm 0.066$ & $7.386 \pm 0.088$ & $7.396 \pm 0.047$ \\
$\mathrm{PaO}_{2}(\mathrm{mmHg})$ & $\mathrm{A}$ & $191.1 \pm 34.1$ & $180.1 \pm 38.7^{\mathrm{a}}$ & $156.4 \pm 30.9^{\mathrm{a}}$ & $186.5 \pm 34.2$ \\
& $\mathrm{~B}$ & $206.8 \pm 45.2$ & $212.1 \pm 50.8^{\mathrm{b}}$ & $208.3 \pm 49.1^{\mathrm{b}}$ & $202.4 \pm 28.6$ \\
$\mathrm{PaCO}_{2}(\mathrm{mmHg})$ & $\mathrm{A}$ & $36.6 \pm 6.0$ & $43.6 \pm 7.7$ & $48.4 \pm 7.6^{\mathrm{a}}$ & $46.6 \pm 7.5^{\mathrm{a}}$ \\
& B & $38.5 \pm 6.3$ & $40.1 \pm 7.0$ & $39.8 \pm 4.4^{\mathrm{b}}$ & $39.3 \pm 2.4$ \\
\hline
\end{tabular}

${ }^{\mathrm{a}} \mathrm{P}<0.05$ compared with the value at $\mathrm{T} 0$, and ${ }^{\mathrm{b}} \mathrm{P}<0.05$ compared with the value in Group $\mathrm{A}$. T0, prior to surgery; $\mathrm{T} 7$, following unilateral femur surgery; T8, following bilateral femur surgery; T10, 240 min following bilateral femur surgery. $\mathrm{PaO}_{2}$, arterial oxygen tension; $\mathrm{PaCO}$, arterial carbon dioxide tension.

pathogenesis of fat embolism, there are currently two hypotheses; the mechanical occlusion theory and the biochemical theory (10). In the most severe case, extensive embolization has been shown to be associated with lung injury and acute respiratory distress syndrome (ARDS) (11). With an enhanced understanding of the disease, an increasing number of studies are currently investigating the inflammatory response in PFE (12-15). In a study on fat embolism in rats, Liu et al demonstrated that serum TNF- $\alpha$, IL-1 $\beta$ and neutrophil elastase levels were increased in pulmonary alveolus irrigating solution, in accordance with an altered lung weight and pulmonary hypertension, and an increased capillary filtration coefficient (14). Blankstein et al identified that the combination of hemorrhagic shock, resuscitation and fat embolism elicited neutrophil activation, infiltration of the alveoli by polymorphonuclear leukocytes and inflammatory cytokine expression, in bronchoalveolar lavage fluid (12). Furthermore, serum IL-6 is considered to be a potential early marker of fat embolism (13). The present study identified that serum TNF- $\alpha$, IL-1 $\beta$ and IL-6 levels were higher than those at the baseline, following the surgical process. These results were consistent with other animal studies $(13,14)$, as well as with certain clinical investigations $(16,17)$. This suggests that these inflammatory cytokines are important during PFE, and subsequently lead to lung injury. Therefore, the early removal of these inflammatory mediators is a potential target of therapy.

Intraoperative cardiovascular deterioration, as a result of pulmonary embolization of bone marrow fat, is a potentially fatal complication during total hip and knee arthroplasty, intramedullary nailing and spine surgery (18). It is important to correct the hemodynamic changes caused by fat embolism. Therefore, comprehensive studies of hemodynamics and early interventions are required. Aebli et al conducted a study in which bone cement was injected into the L1 vertebral bodies of six sheep, and demonstrated that following the injection of the bone cement, there were reflective reductions in the HR and 
A

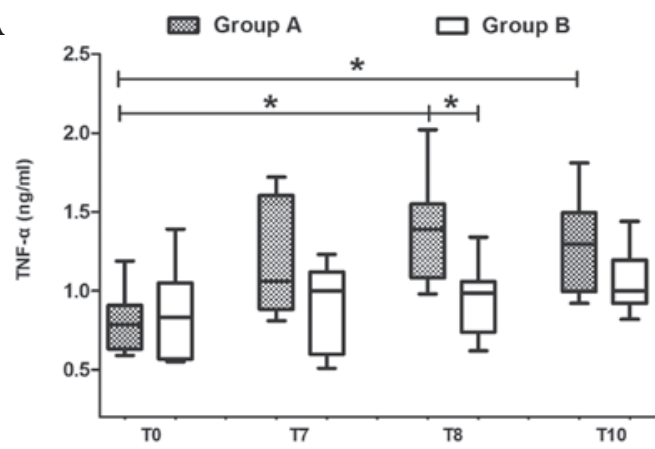

B

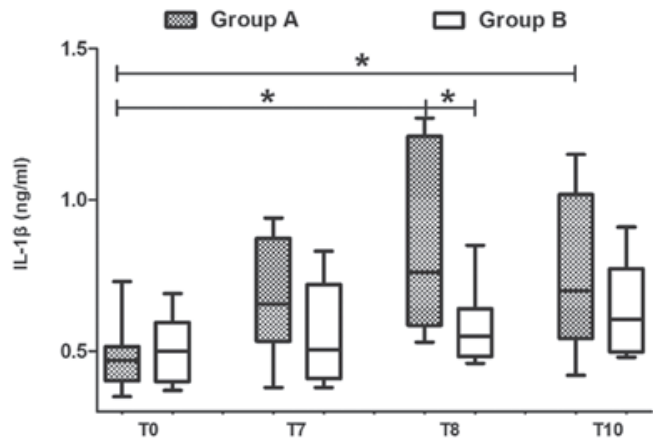

C

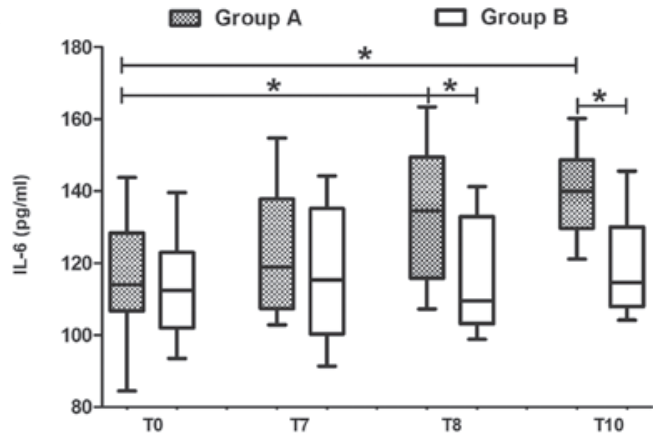

Figure 4. Alterations in the cytokine levels at various time points during and following intramedullary surgery in Groups A and B. Changes in the plasma concentration of (A) tumor necrosis factor- $\alpha$ (TNF- $\alpha)$, (B) interleukin-1 $\beta$ (IL-1ß) and (C) IL-6. ${ }^{*} \mathrm{P}<0.05$ between groups $\mathrm{A}$ and $\mathrm{B}$. T0, prior to surgery; $\mathrm{T} 7$, following unilateral femur surgery; $\mathrm{T} 8$, following bilateral femur surgery; T10, 240 min following bilateral femur surgery.

arterial pressure, and that the second reduction in the arterial pressure was caused by secondary PFE (19). Similar results were observed in the present study. Significant increases in the HR, CVP, MPAP, PCWP and EVLW and a significant reduction in the MAP occurred immediately following the reaming and bone cement infusion processes. It was thought that the changes were associated with the intramedullary procedures, leading to the release of bone marrow particles and the formation of microthrombi in the pulmonary artery. This was confirmed by Oil Red O staining of pulmonary sections from the current study animals. Wheelwright et al also concluded that pulmonary fat and marrow embolism were key factors of cardiorespiratory and hemodynamic instability, following canal pressurization (20). It is likely that the methylmethacrylate monomer, acting as a systemic vasodilator, is also a factor during this procedure (21).

Pathological examinations are able to reveal the presence of alveolar edema and hemorrhage with multiple fat droplet depositions and fibrin thrombi; however, chest radiography is unable to locate these during the early stages of fat embolism (16). EVLW is a useful measure of the accumulation of parenchymal lung edema, and there has previously been shown to be a significant correlation between EVLW measurements indexed to all body weights and the severity of lung injury in patients with ARDS (22). In the present study, the EVLW levels remained continuously high from the completion of the intramedullary procedure to $240 \mathrm{~min}$ following the bilateral femur surgery. Furthermore, following the bilateral femoral intramedullary procedure, there was a reduction in the $\mathrm{pH}$ and the $\mathrm{PaO}_{2}$, and an increase in the $\mathrm{PaCO}_{2}$. The cytokine levels, specifically those of TNF- $\alpha$, IL- $1 \beta$ and IL-6, were also higher compared with those prior to surgery. Thus, the intramedullary surgery resulted in the formation of PFE and the release of inflammatory cytokines. This increased the permeability of the pulmonary microcirculation and the EVLW, which subsequently resulted in a reduction in the $\mathrm{PaO}_{2}$ and an increase in the $\mathrm{PaCO}_{2}$. The $\mathrm{PaO}_{2}$ and the $\mathrm{PaCO}_{2}$ were correlated with the EVLW $(\mathrm{P}<0.05)$, indicating that EVLW, as an independent index, may be valuable in the evaluation of pulmonary edema during the early stages of PFE.

The present study employed tracheal intubation, general anesthesia and controlled mechanical ventilation procedures. The basic settings of the ventilator remained unchanged, and therefore minimized the influence of other factors on respiration. The pathological results indicated that intramedullary procedures were able to evoke PFE, which mainly resulted in a reduced $\mathrm{PaO}_{2}$ and an increased $\mathrm{PaCO}_{2}$ during surgery. Similar results have been identified in other studies $(6,23)$. A potential reason for the induction of PFE may be that a fat embolus entered the lung, which decreased the blood perfusion and exchange area, and subsequently increased the dead space area. Alternatively, in this study, as the basic settings of the ventilator remained unchanged, the pulmonary edema caused by fat embolism and the release of inflammatory cytokines may have resulted in an increased airway pressure and a decreased tidal volume. Bilateral procedures carry higher risks compared with unilateral ones.

Although the results of the present study indicated that the pulmonary tissue sections of all animals in Group A exhibited fat particles, the present embolism model did not lead to severe changes in clinical symptoms. Arterial blood gases and certain hemodynamic parameters, such as HR and MAP, improved 240 min following the bilateral procedures (T10). These results were in accordance with the fact that the majority of intramedullary reaming processes do not induce FES (24). Discordance between the clinical and experimental diagnosis of fat embolism, or ARDS, was mainly due to pulmonary infiltration $(25,26)$ or compensation of the patient and the size of the fat embolus (27). In a clinical study, Kim revealed that $65 \%$ of patients who underwent bilateral knee joint replacement and $46 \%$ of patients who underwent unilateral knee joint replacement demonstrated positive Oil Red O staining of fat particles in their blood (28). Pitto et al studied patients with a femoral neck fracture who received cemented total hip joint replacement and identified, using transesophageal echocardiography (TEE), that $95 \%$ of the patients had fat embolism presentations (29). The aforementioned studies suggest a high prevalence of PFE induced by intramedullary procedures, with PFE as a potential complication of such procedures. The 
treatment options, such as clofibrate, dextran-40, ethyl alcohol, heparin and aspirin, have been evaluated previously and shown to cause no significant changes in the clinical outcomes of PFE and FES $(27,30)$. Strict monitoring of the hemodynamics during surgery is essential if a patient has high risk factors, such as multiple injuries and infection, in the clinical setting. In the case of continuously abnormal hemodynamics, an early intervention is required to prevent fat embolism from developing into FES.

There were several limitations to this study. In Group B, the surgery was conducted without opening the medullary cavity. Therefore, it was not possible to compare the hemodynamic parameters of the two groups during the surgical procedure in the medullary cavity, such as medullary reaming and bone cement injection. However, comparisons between Groups A and $\mathrm{B}$ demonstrated that the animals in Group A were effective models in which to evaluate PFE through postmortem pulmonary tissues. Furthermore, the model allowed for the creation of fat embolism and the tracking of the changes in cytokine levels and arterial blood gas parameters during the surgical procedure. Another limitation of this study was that the dogs were kept alive for only four hours following surgery, and the risk factors were determined during the early stages of PFE. Therefore, it was not possible to draw conclusions regarding the development of fat embolism and inflammation over a period of several days following the procedure. The focus was on the initial impact of PFE, during or following the surgery. Therefore, further experimental studies in animals are required to investigate these possible events.

In conclusion, femoral intramedullary surgery may induce PFE and affect the hemodynamics and arterial blood gases, for example, by reducing the $\mathrm{PaO}_{2}$ and increasing the $\mathrm{PaCO}_{2}$. The EVLW was demonstrated to correlate with the $\mathrm{PaO}_{2}$ and the $\mathrm{PaCO}_{2}$, and may serve as a predictor for the development of FES. Positive Oil Red O staining in pulmonary tissue sections was observed in animals that received intramedullary surgery, indicating the high prevalence of PFE induced by such a surgical procedure. The serum concentrations of TNF- $\alpha$, IL-1 $\beta$ and IL-6 increased following the intramedullary surgery, and these cytokines may therefore be a risk factor for the development of FES.

\section{References}

1. Stein PD, Yaekoub AY, Matta F and Kleerekoper M: Fat embolism syndrome. Am J Med Sci 336: 472-477, 2008.

2. Eriksson EA, Pellegrini DC, Vanderkolk WE, Minshall CT, Fakhry SM and Cohle SD: Incidence of pulmonary fat embolism at autopsy: an undiagnosed epidemic. J Trauma 71: 312-315, 2011.

3. Ereth MH, Weber JG, Abel MD, et al: Cemented versus noncemented total hip arthroplasty - embolism, hemodynamics, and intrapulmonary shunting. Mayo Clin Proc 67: 1066-1074, 1992.

4. Bolliger SA, Muehlematter K, Thali MJ and Ampanozi G: Correlation of fat embolism severity and subcutaneous fatty tissue crushing and bone fractures. Int J Legal Med 125: 453-458, 2011.

5. Krebs J, Ferguson SJ, Hoerstrup SP, Goss BG, Haeberli A and Aebli N: Influence of bone marrow fat embolism on coagulation activation in an ovine model of vertebroplasty. J Bone Joint Surg Am 90: 349-356, 2008.

6. Blankstein M, Byrick RJ, Richards RR, Mullen JB, Zdero R and Schemitsch EH: Pathophysiology of fat embolism: a rabbit model. J Orthop Trauma 25: 674-680, 2011.
7. McIff TE, Poisner AM, Herndon B, Lankachandra K, Molteni A and Adler F: Mitigating effects of captopril and losartan on lung histopathology in a rat model of fat embolism. J Trauma 70: 1186-1191, 2011.

8. Liu DD, Hsieh NK and Chen HI: Histopathological and biochemical changes following fat embolism with administration of corn oil micelles: a new animal model for fat embolism syndrome. J Bone Joint Surg Br 90: 1517-1521, 2008.

9. McIff TE, Poisner AM, Herndon B, et al: Fat embolism: evolution of histopathological changes in the rat lung. J Orthop Res 28: 191-197, 2010.

10. Shaikh N: Emergency management of fat embolism syndrome. J Emerg Trauma Shock 2: 29-33, 2009.

11. Fong DL, Murnane RD, Hotchkiss CE, Green DJ and Hukkanen RR: Pulmonary embolization of fat and bone marrow in cynomolgus Macaques (Macaca fascicularis). Comp Med 61: 86-91, 2011.

12. Blankstein M,Byrick RJ,Nakane M,etal: Amplifiedinflammatory response to sequential hemorrhage, resuscitation, and pulmonary fat embolism: an animal study. J Bone Joint Surg Am 92: 149-161, 2010.

13. Yoga R, Theis JC, Walton M and Sutherland W: Interleukin-6 as an early marker for fat embolism. J Orthop Surg Res 4: 18, 2009.

14. Liu DD, Kao SJ and Chen HI: N-acetylcysteine attenuates acute lung injury induced by fat embolism. Crit Care Med 36: 565-571, 2008.

15. Kao SJ and Chen HI: Nitric oxide mediates acute lung injury caused by fat embolism in isolated rat's lungs. J Trauma 64 : 462-469, 2008

16. Kao SJ, Yeh DY and Chen HI: Clinical and pathological features of fat embolism with acute respiratory distress syndrome. Clin Sci (Lond) 113: 279-285, 2007.

17. Hsu YH, Kao SJ, Lee RP and Chen HI: Acute pulmonary oedema: Rare causes and possible mechanisms. Clin Sci (Lond) 104: 259-264, 2003.

18. Krebs J, Ferguson SJ, Nuss K, et al: Sildenafil prevents cardiovascular changes after bone marrow fat embolization in sheep. Anesthesiology 107: 75-81, 2007.

19. Aebli N, Krebs J, Davis G, Walton M, Williams MJ and Theis JC: Fat embolism and acute hypotension during vertebroplasty: an experimental study in sheep. Spine (Phila Pa 1976) 27: 460-466, 2002.

20. Wheelwright EF, Byrick RJ, Wigglesworth DF, et al: Hypotension during cemented arthroplasty. Relationship to cardiac output and fat embolism. J Bone Joint Surg Br 75: 715-723, 1993.

21. Peebles DJ, Ellis RH, Stride SD and Simpson BR: Cardiovascular effects of methylmethacrylate cement. Br Med J 1: 349-351, 1972.

22. Berkowitz DM, Danai PA, Eaton S, Moss M and Martin GS: Accurate characterization of extravascular lung water in acute respiratory distress syndrome. Crit Care Med 36: 1803-1809, 2008.

23. Whelan DB, Byrick RJ, Mazer CD, et al: Posttraumatic lung injury after pulmonary contusion and fat embolism: factors determining abnormal gas exchange. J Trauma 69: 512-518, 2010.

24. Kaufmann TJ, Jensen ME, Ford G, Gill LL, Marx WF and Kallmes DF: Cardiovascular effects of polymethylmethacrylate use in percutaneous vertebroplasty. AJNR Am J Neuroradiol 23: 601-604, 2002.

25. Murphy P, Edelist G, Byrick RJ, Kay JC and Mullen JB: Relationship of fat embolism to haemodynamic and echocardiographic changes during cemented arthroplasty. Can J Anaesth 44: 1293-1300, 1997.

26. Lehnert BE: Pulmonary and thoracic macrophage subpopulations and clearance of particles from the lung. Environ Health Perspect 97: 17-46, 1992.

27. Mellor A and Soni N: Fat embolism. Anaesthesia 56: 145-154, 2001.

28. Kim YH: Incidence of fat embolism syndrome after cemented or cementless bilateral simultaneous and unilateral total knee arthroplasty. J Arthroplasty 16: 730-739, 2001.

29. Pitto RP, Blunk J and Kössler M: Transesophageal echocardiography and clinical features of fat embolism during cemented total hip arthroplasty. A randomized study in patients with a femoral neck fracture. Arch Orthop Trauma Surg 120: 53-58, 2000.

30. Taviloglu K and Yanar H: Fat embolism syndrome. Surg Today 37: $5-8,2007$ 